\title{
Analytical Method Using Scaled Hypersphere Search for High-dimensional Metadynamics Simulations
}

\author{
Yuki Mitsuta* and Yasuteru Shigeta* \\ Center for Computational Sciences, University of Tsukuba 1-1-1 Tennodai, Tsukuba, Ibaraki, \\ Japan, 305-8577
}

Email: mitsutay@ccs.tsukuba.ac.jp,shigeta@ccs.tsukuba.ac.jp 

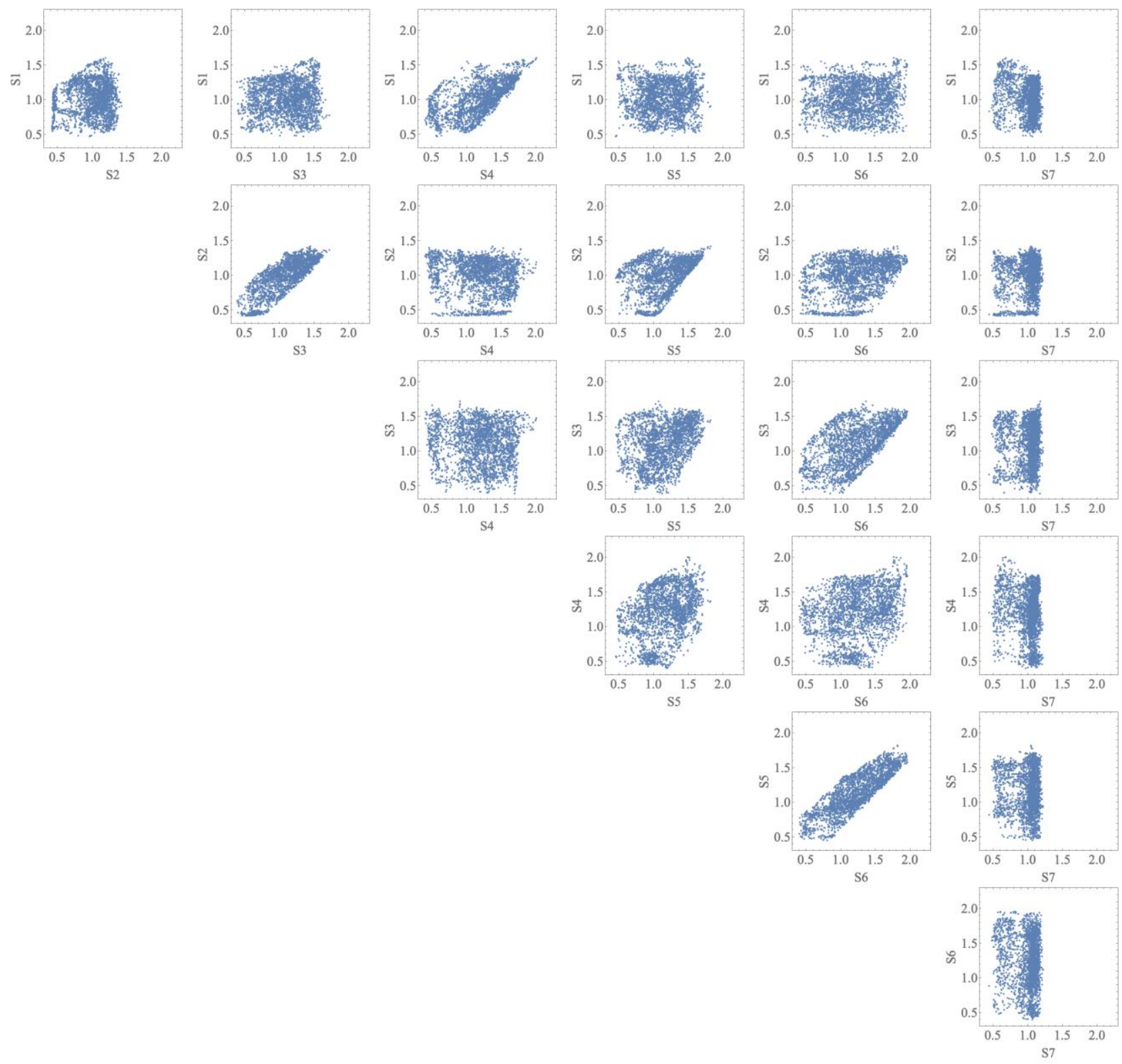

Figure S1. Plots of the coordination of the EQ and TS points on two CVs. 


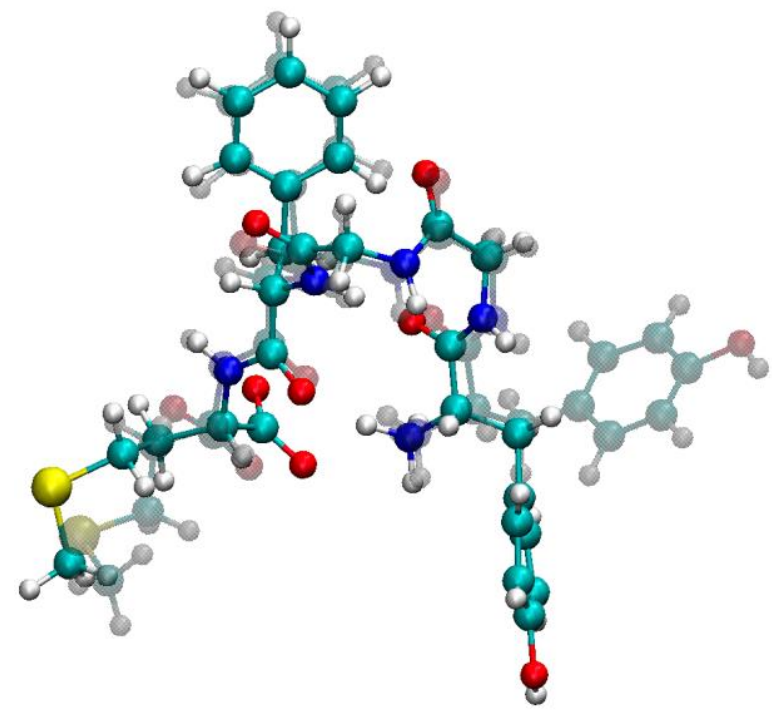

Figure S2. The EQ structures of EQ3 (solid color) and EQ7 (shadow color).

Table S1. Dihedral angles of the Ramachandran plot for each EQ point.

\begin{tabular}{|lllllll|}
\hline & $\begin{array}{l}\phi_{\mathrm{GLY} 2} \\
(\text { degree })\end{array}$ & $\begin{array}{l}\psi_{\mathrm{GLY} 2} \\
(\text { degree })\end{array}$ & $\begin{array}{l}\phi_{\mathrm{GLY} 3} \\
(\text { degree })\end{array}$ & $\begin{array}{l}\psi_{\mathrm{GLY} 3} \\
(\text { degree })\end{array}$ & $\begin{array}{l}\phi_{\mathrm{PHE} 4} \\
(\text { degree })\end{array}$ & $\begin{array}{l}\psi_{\mathrm{PHE} 4} \\
\text { (degree) }\end{array}$ \\
\hline EQ1 & -47.2 & 139.2 & 84.6 & 4.3 & 81.3 & 44.8 \\
EQ2 & -75.9 & -17.4 & -89.5 & 21.1 & -130.0 & 7.1 \\
EQ3 & 79.7 & 16.6 & 75.5 & 19.9 & -132.6 & 169.1 \\
EQ4 & -51.7 & 160.8 & -74.4 & -51.7 & -94.7 & 16.6 \\
EQ5 & 63.5 & 32.6 & 105.2 & 63.5 & -155.5 & 153.4 \\
EQ6 & -73.2 & -23.9 & -109.0 & -73.2 & -62.6 & -36.5 \\
EQ8 & 79.4 & 1.4 & 100.1 & 79.4 & -121.6 & 157.9 \\
EQ9 & 87.2 & 8.2 & 81.9 & 2.2 & -92.1 & -15.6 \\
EQ10 & 82.0 & -6.7 & 80.6 & 6.3 & -104.8 & -11.6 \\
EQ11 & 84.3 & 16.7 & 87.8 & 9.0 & -151.3 & 155.0 \\
EQ12 & 80.7 & 15.6 & 95.5 & -0.1 & -133.8 & 144.6 \\
EQ13 & 67.4 & 17.2 & 85.7 & -7.1 & -94.6 & -39.0 \\
EQ14 & -75.6 & -175.9 & -87.8 & -4.8 & -119.1 & 1.6 \\
\hline
\end{tabular}


Table S2. Kinetic Matrix of the contracted-FERN of the RCMC analysis until the maximum rate constant as $k_{\max }=0.01 \mathrm{ps}{ }^{-1}=$ $10 \mathrm{~ns}^{-1}$. The direction is from SS of the column to that of the row. The unit of each kinetic is ns ${ }^{-1} . k=0.000$ means that there is not connection between the two points.

\begin{tabular}{|c|cccccccccccc|}
\hline from & $\mathrm{SS}_{\mathrm{a}}$ & $\mathrm{SS}_{\mathrm{b}}$ & $\mathrm{SS}_{\mathrm{c}}$ & $\mathrm{SS}_{\mathrm{d}}$ & $\mathrm{SS}_{\mathrm{e}}$ & $\mathrm{SS}_{\mathrm{f}}$ & $\mathrm{SS}_{\mathrm{g}}$ & $\mathrm{SS}_{\mathrm{h}}$ & $\mathrm{SS}_{\mathrm{i}}$ & $\mathrm{SS}_{\mathrm{j}}$ & $\mathrm{SS}_{\mathrm{k}}$ & $\mathrm{SS}_{1}$ \\
\hline $\mathrm{SS}_{\mathrm{a}}$ & - & 0.426 & 0.447 & 0.007 & 0.016 & 1.028 & 0.295 & 0.103 & 0.000 & 0.019 & 0.000 & 0.081 \\
$\mathrm{SS}_{\mathrm{b}}$ & 1.111 & - & 0.312 & 0.494 & 0.532 & 0.850 & 0.030 & 0.395 & 0.005 & 0.007 & 0.019 & 0.698 \\
$\mathrm{SS}_{\mathrm{c}}$ & 2.335 & 0.625 & - & 0.005 & 0.173 & 9.066 & 0.908 & 0.033 & 0.084 & 0.001 & 0.000 & 2.922 \\
$\mathrm{SS}_{\mathrm{d}}$ & 0.021 & 0.591 & 0.003 & - & 0.124 & 0.004 & 0.000 & 1.467 & 0.000 & 0.000 & 0.000 & 0.032 \\
$\mathrm{SS}_{\mathrm{e}}$ & 0.065 & 0.853 & 0.138 & 0.167 & - & 0.103 & 0.015 & 0.334 & 0.008 & 0.000 & 0.285 & 2.238 \\
$\mathrm{SS}_{\mathrm{f}}$ & 5.348 & 1.695 & 9.036 & 0.006 & 0.129 & - & 0.309 & 0.072 & 0.003 & 0.002 & 0.000 & 1.332 \\
$\mathrm{SS}_{\mathrm{g}}$ & 2.042 & 0.080 & 1.204 & 0.001 & 0.025 & 0.411 & - & 0.006 & 0.013 & 0.001 & 0.000 & 0.171 \\
$\mathrm{SS}_{\mathrm{h}}$ & 0.885 & 1.296 & 0.053 & 4.028 & 0.684 & 0.118 & 0.007 & - & 0.001 & 0.005 & 0.000 & 0.179 \\
$\mathrm{SS}_{\mathrm{i}}$ & 0.199 & 0.765 & 6.822 & 0.017 & 0.770 & 0.262 & 0.775 & 0.033 & - & 0.000 & 0.000 & 4.303 \\
$\mathrm{SS}_{\mathrm{j}}$ & 3.723 & 0.557 & 0.041 & 0.004 & 0.002 & 0.095 & 0.031 & 0.111 & 0.000 & - & 0.000 & 0.008 \\
$\mathrm{SS}_{\mathrm{k}}$ & 0.000 & 0.198 & 0.000 & 0.000 & 1.818 & 0.000 & 0.000 & 0.000 & 0.000 & 0.000 & - & 0.000 \\
$\mathrm{SS}_{\mathrm{l}}$ & 1.243 & 4.110 & 8.604 & 0.155 & 8.230 & 3.934 & 0.379 & 0.321 & 0.156 & 0.001 & 0.000 & - \\
\hline
\end{tabular}




\section{Parameters for the SHS Calculation}

Table S1 summarizes the parameters chosen in the study. The optimization of the NewtonRaphson method and the dimer approach were performed using the threshold of the gradient.

In the SHS calculation, it is important to firstly calculate the scaled hypersphere surface (SHsurface). To reduce the structure of the harmonic potential, we selected the parameter of length between an EQ point and the initial SH-surface along the harmonic potential hardest mode. The stepsize of uphill walking of the SH-surfaces was calculated using the length of the hardest mode.

In addition, the threshold to identify the same EQ or TS points was prepared, which should be larger than the optimization error. In our calculation, the dimer method was used, which was improved by Kästner and Sherwood. ${ }^{1}$ In this method, two parameters are considered, i.e., the length of dimer and the threshold of rotation minimizing $\left|\phi_{1}\right|$.

Calculating the gradient down from a TS point, there is an MFEP on the TS point along the imaginary frequency direction $v_{1}$, which is the normalized eigenvector with a negative eigenvalue:

$$
S_{ \pm 1}=S_{0} \pm v_{1} \cdot \Delta S_{0}
$$

where $S_{0}$ is the $\mathrm{CV}$ of the TS point and $\Delta s_{0}$ denotes the parameter of the first step. After calculating $S_{ \pm 1}$, the subsequent step involves parameter $\Delta s$;

$$
S_{m \pm 1}=S_{m}-\frac{g\left(S_{m}\right)}{\left|g\left(S_{m}\right)\right|} \cdot \Delta s
$$

Note that $\Delta s_{0}$ should be larger than $\Delta s .^{2}$ 
Table S3. Parameters for the SHS calculation.

\begin{tabular}{|c|c|c|c|}
\hline parameter name & meanings & Styblinski-Tang & met-enkephalin \\
\hline threshold & $\begin{array}{l}\text { Threshold of gradient in the } \\
\text { optimizations in the Newton- } \\
\text { Raphson method and the dimer } \\
\text { method. }\end{array}$ & 0.1 & 0.1 \\
\hline sameEQthreshold & $\begin{array}{l}\text { Threshold to identify same EQ or TS } \\
\text { points. }\end{array}$ & 0.05 & 0.05 \\
\hline IOEsphereA_initial & $\begin{array}{l}\text { Length to calculate the potential of } \\
\text { initial SH surface. }\end{array}$ & 0.2 & 0.02 \\
\hline IOEsphereA_dist & $\begin{array}{l}\text { Length to calculate the stepsize of } \\
\text { SH surfaces. }\end{array}$ & 0.05 & 0.005 \\
\hline Ddimer & $\begin{array}{l}\text { Distance of dimer in the dimer } \\
\text { method. }\end{array}$ & 0.05 & 0.05 \\
\hline phitol & $\begin{array}{l}\text { Threshold of }\left|\phi_{1}\right| \text { in the dimer } \\
\text { method. }\end{array}$ & 0.08 & 0.08 \\
\hline deltas 0 & $\Delta s_{0}$ in equation ( $\left.\mathrm{S} 1\right)$ & 0.01 & 0.01 \\
\hline deltas & $\Delta s$ in equation ( $\mathrm{S} 2)$. & 0.005 & 0.005 \\
\hline
\end{tabular}




\section{Test Calculation for a Two-dimensional Example}

In the present section, a test calculation for a two-dimensional toy example is demonstrated using the following function:

$$
V(x, y)=\sin (\pi x) \sin (\pi y) \text {. }
$$

This function has a large number of global minima at $(x, y)=(n-0.5, m+0.5)$, where $n$ and $m$ are integers. For instance, $(0.5,-0.5)$ is a global minimum surrounded by 4 saddle states at $(0,0),(1,0),(0,-1)$, and $(1,-1)$. Notably, the saddle states are not located along the frequency directions of the minimum point. In Figure S3, the SHS searching from $(0.5,-0.5)$ is shown. The black lines are plotted along ADDs of each SH-surface, which is different from the frequency directions, and the 4 saddle states are found simultaneously.

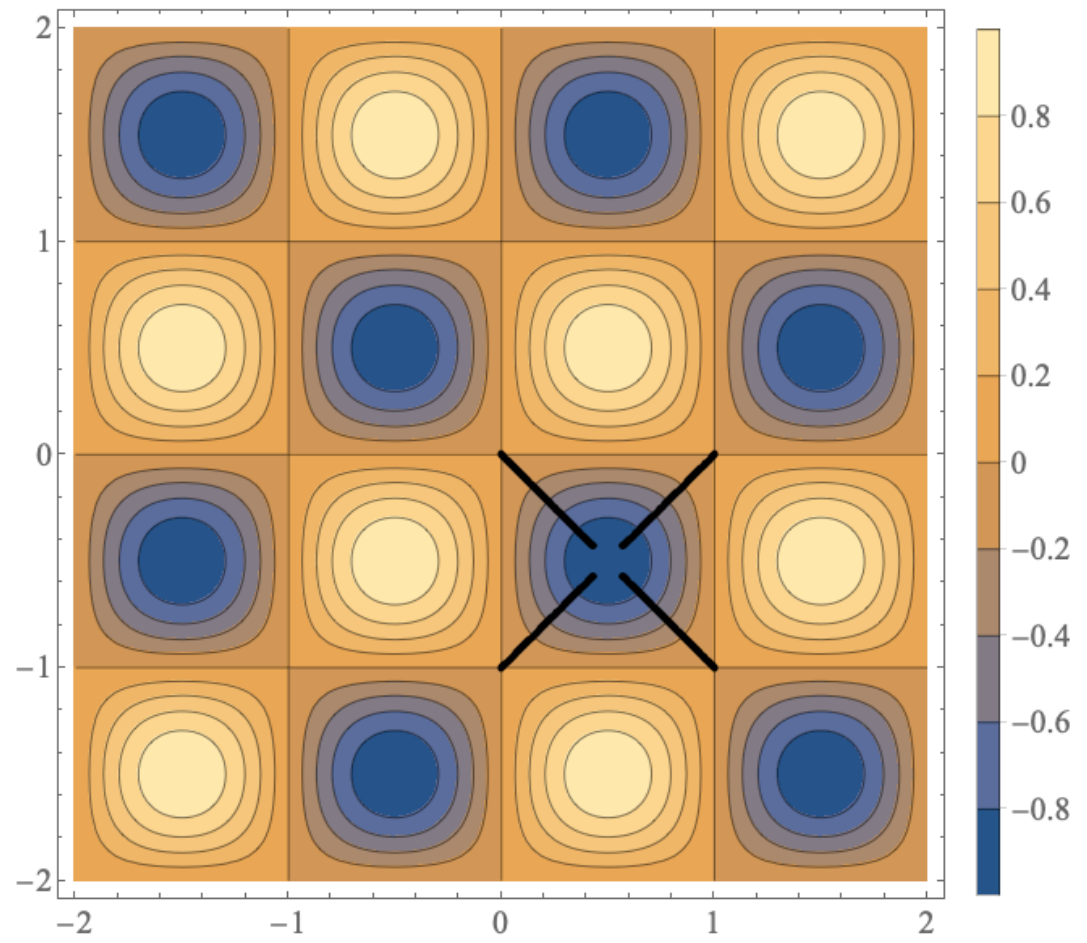

Figure S3. Contour plot of equation (S3) and the SHS calculated from the equation point of $(0.5,-0.5)$. The black lines indicate the paths of ADDs until the saddle points are found. 


\section{Estimation of Frequency Factors}

In equation (6), the frequency factor $A$ is a parameter, which depends on each MFEP path. Moreover, the velocity depends on CVs, and theoretical estimation of $\mathrm{A}$ is difficult. To calculate it using the result of the performed simulations, we used the trajectory of metadynamics. After the metadynamics simulation, the potential of FEL with the bias potential is approximately flatten, which means that $F_{T S}-F_{a} \approx 0$. The velocity of the simulation with the bias potential is approximate to the frequency factor. For example, the METAGUI tool ${ }^{3}$ can be used to calculate the velocity using grid spacing ${ }^{4}$; however, it is difficult to apply it for high-dimensional CVs due to the limitation of computational costs. In the present study we analyzed the trajectory directory and collected the velocity of the nearest points.

The frequency factors were calculated according to the following steps:

1. The MD simulation was performed with the bias potential after the metadynamics simulation. In the present study, the multiple walker MTD without adding new bias potentials was performed in $10 \mathrm{~ns}$ for each of the 10 walkers, affording a $100 \mathrm{~ns}$ trajectory.

2. The trajectory was analyzed and the velocities on CVs of the sampling were obtained. Because the grid spacing was difficult, we constructed the velocities along the trajectory of the MD simulation. In this step, we focused on the microstate separated by the distance of $0.1 \mathrm{~nm}$ on the CVs. The initial point on the trajectory was defined. The time was summed until separation over $0.1 \mathrm{~nm}$ was detected and the last point was defined. The velocity was calculated as the time divided by the length between the initial point and the last point. The last point was defined as a new initial point and the calculation was iterated. We attempted changing the distance of the microstate to $0.2 \mathrm{~nm}$ and $0.5 \mathrm{~nm}$; however, the qualitative results were not affected. 
3. The distance between MFEP and the sampled points was compared, and 50 velocities of the nearest points were collected. The average of the velocities was the velocity on the CVs along the MFEP.

4. The length of the MFEP was divided by the velocity on the CVs. The velocity between two EQ points was calculated. The velocity was approximately equal to the frequency factor of the velocity along the MFEP.

\section{References}

[1] J. Kästner and P. Sherwood, "Superlinearly converging dimer method for transition state search," J. Chem. Phys., vol. 128, no. 1, p. 014106, 2008.

[2] M. U. Bohner and J. Kästner, "An algorithm to find minimum free-energy paths using umbrella integration," J. Chem. Phys., vol. 137, no. 3, pp. 0-6, 2012.

[3] X. Biarnés, F. Pietrucci, F. Marinelli, and A. Laio, "METAGUI. A VMD interface for analyzing metadynamics and molecular dynamics simulations," Comput. Phys. Commun., vol. 183, no. 1, pp. 203-211, 2012.

[4] F. Noé, I. Horenko, C. Schütte, and J. C. Smith, "Hierarchical analysis of conformational dynamics in biomolecules: Transition networks of metastable states," J. Chem. Phys., vol. 126, no. 15, 2007. 